\title{
Axillary Lymph Node to Primary Breast Tumor Standardized Uptake Value Ratio from FDG-PET/CT Imaging for Predicting the Necessity for Nodal Dissection in Primary Breast Tumors
}

\author{
Han-kyul Shin, Min Kyoon Kim, Sung Jun Park, Ju Won Seok ${ }^{1}$, Hee-Chul Shin \\ Departments of Surgery and ${ }^{1}$ Nuclear Medicine, Chung-Ang University College of Medicine, Seoul, Korea
}

Purpose: Accurate preoperative detection by radiologic assessment is necessary to specifically identify patients with at least three positive nodes, who can directly undergo axillary lymph node (ALN) dissection, and avoid unnecessary surgical procedures. We evaluated the usefulness of the standardized uptake value (SUV) ratio of ALN in primary breast tumor, using ${ }^{18} \mathrm{~F}$-fluorodeoxyglucose positron emission tomography (FDG-PET)/computed tomography (CT) and magnetic resonance imaging (MRI) to predict the necessity of ALN dissection during breast cancer surgery. Methods: In this retrospective study we enrolled 316 consecutive patients with invasive breast cancer. The SUV ratio of ALN to primary breast tumor uptake was calculated. Optimal cutoff values were determined by receiver operating characteristic curve analysis for predicting the presence of $\geq 3$ ALN metastases. Diagnostic performance of FDG-PET and MRI features for the prediction of $\geq 3$ ALN metastases were determined by sensitivity, specificity, and diagnostic odds ratio (DOR). A subgroup analysis for FDG-avid tumors was also performed. Results: Of the 316 patients, 36 (11.4\%) showed involvement of $\geq 3$ ALNs, with 101 (32\%) having at least one metastatic lymph node. Axillary ${ }^{18} \mathrm{~F}$-FDG uptake was positive in 75 patients (23.7\%), and the optimal ratio of maximum SUV of axillary lymph node and primary tumor for determining ALN dissection was 0.3 . MRI scans revealed suspicious ALN involvement in 147 patients (46.6\%). The sensitivity and specificity of MRI detection were $88.9 \%$ and $56.2 \%$, respectively, while for SUV $\mathrm{LNT}_{\mathrm{T}}$ ratio, they were $69.4 \%$ and $86.8 \%$, respectively. DOR values for MRI and SUV $V_{\text {LNT }}$ ratio were 10.37 and 9.7, respectively. The area under the curve (AUC) was improved to 0.896 (95\% confidence interval [Cl], 0.817-0.975) for the SUV $V_{L N T}$ ratio in patients with FDG-avid primary tumors (FDG $\geq 3.9, n=108$ ), but the MRI AUC was worsened $(0.681 ; 95 \% \mathrm{Cl}, 0.569-0.793)$. The DOR, sensitivity, and specificity for the SUVLNT ratio of FDG-avid cancers were 25.68, $89.0 \%$ and $86.0 \%$, respectively. Conclusion: SUV LNT ratio outperformed MRI features in predicting the need for ALN dissection in FDG-avid primary breast cancer. PET/CT may be a potential noninvasive diagnostic technique for identifying the presence of $\geq 3$ ALN metastases.

Key Words: Breast neoplasms, Neoadjuvant chemotherapy, Clinical response

\section{INTRODUCTION}

The American College of Surgeons Oncology Group Z0011 trial indicated that complete axillary lymph node dissection (ALND) did not improve survival in women with clinical T1-T2 tumors and 1 to 2 involved axillary nodes, who underwent lumpectomy with radiation therapy, followed by systemic therapy [1]. As the treatment for both the axillary lymph nodes (ALNs) and primary breast tumors evolve, predicting the status of the ALNs has become increasingly important. However, intraoperative frozen sections of sentinel lymph node (SLNs)

\section{Correspondence: Hee-Chul Shin}

Breast Clinic, Department of Surgery, Chung-Ang University Hospital, ChungAng University College of Medicine, 102 Heukseok-ro, Dongjak-gu, Seoul 06973, Korea

Tel: +82-2-6299-1565, Fax: +82-2-6299-1548, E-mail: dradam77@naver.com Received: Oct 10, 2017 Revised: Nov 19, 2017 Accepted:Dec 6, 2017 or imaging-guided ALN biopsy still show a high false-negative rate of approximately $14 \%$ to $43 \%[2,3]$

To appropriately determine the necessity of ALND, evaluation of the axillary tumor burden is critical. Ultrasonography, computed tomography $(\mathrm{CT})$, or magnetic resonance imaging (MRI) primarily depend on lymph node (LN) size or morphology for differentiation between metastatic and nonmetastatic status. Therefore, normal- and small-size metastatic LNs can easily remain undetected. Furthermore, the variable morphologic characteristics of LNs also reduce diagnostic accuracy owing to the overlapping features of metastatic and nonmetastatic LNs [4].

Following the advances in preoperative imaging techniques, some investigators have described the usefulness of ${ }^{18} \mathrm{~F}$-fluorodeoxyglucose positron emission tomography (FDG-PET)/CT, a metabolic imaging modality, for ALN staging in breast cancer $[5,6]$. However, the report- 
ed sensitivity and specificity of FDG-PET/CT for ALN metastasis are low at $48 \%$ to $58 \%$ and $84 \%$ to $95 \%$, respectively $[7,8]$.

We hypothesized that, although it would be difficult to distinguish involvement of a single LN, it may be possible to use FDG-PET/CT to predict high LN tumor burden, defined as involvement of $\geq 3$ ALNs. In the present study, we evaluated the usefulness of the standardized uptake value (SUV) ratio of ALN to primary breast tumor, as determined by FDG-PET/CT, as compared to MRI, for predicting the necessity of ALND in breast cancer surgery.

\section{METHODS}

\section{Study design}

The electronic medical records of 429 consecutive patients with invasive breast cancer, treated at Chung-Ang University Hospital between January 2012 and December 2016, were retrospectively analyzed. Patients were included if they (1) had histopathologically confirmed invasive breast cancer and (2) undergone pretreatment breast MRI and FDG-PET/CT imaging. Patients were excluded according to the following criteria: (1) any prior treatment for primary breast cancer; (2) ductal carcinoma in situ; or (3) breast cancer type other than adenocarcinoma. Finally, 316 consecutive patients were enrolled in the study following application of the inclusion/exclusion criteria. Patients underwent ALND if there was at least one metastatic sentinel LN from the frozen biopsy result collected intraoperatively. This study was reviewed and approved by the Institutional Review Board of Chung-Ang University Hospital (IRB number: 1711-011-16120), and the informed consent was waived.

\section{Radiologic analyses}

PET/CT scans were performed on a Biograph mCT/128 PET/CT scanner (Siemens Medical Solutions, Hoffman Estates, USA). All patients fasted for at least 6 hours before being administered FDG (4.81 MBq/kg of body weight, intravenously) and had blood glucose levels less than $150 \mathrm{mg} / \mathrm{dL}$. Whole-body PET/CT was performed from the skull base to the proximal thigh in the supine position. PET/CT imaging began 60 minutes after FDG injection. Ellipsoid volumes of interest that included the entire breast tumor and ALNs were segmented, and maximum SUVs of the breast tumor $\left(\mathrm{SUV}_{\mathrm{T}}\right)$ and ALNs $\left(\mathrm{SUV}_{\mathrm{LN}}\right)$ were measured on each PET/CT data set. $\mathrm{SUV}_{\mathrm{LN}}$ to $S U \mathrm{~V}_{\mathrm{T}}$ ra- tios $\left(\mathrm{SUV}_{\mathrm{LN} / \mathrm{T}}\right)$ were then calculated.

MRI scans were acquired on a 3.0 Tesla scanner (Achieva; Philips Medical Systems, Best, the Netherlands), using a 7-channel dedicated breast coil system with the patient in the prone position. MRI was performed as reported in a previous study [9].

Evaluation of ALN status was performed using visual analysis on FDG-PET. Positivity was defined as a perceptibly higher metabolism of ALN compared to the normal background soft tissue [10]. MRI scans were defined to be positive for ALN metastasis if the characteristics of LNs included the following: $>10 \mathrm{~mm}$ in size, rounded shape, eccentric cortical hypertrophy, or abnormal enhancement on T1weighted images.

Table 1. Demographic characteristics of patients included in the study $(n=316)$

\begin{tabular}{|c|c|}
\hline Characteristic & No. (\%) \\
\hline Age $(y r)^{*}$ & $55.1 \pm 11.8(28-86)$ \\
\hline BMI $\left(\mathrm{kg} / \mathrm{m}^{2}\right)^{*}$ & $24.2 \pm 3.8(16-44.2)$ \\
\hline \multicolumn{2}{|l|}{ Histologic type } \\
\hline IDC & $269(85.1)$ \\
\hline ILC & $10(3.2)$ \\
\hline Mucinous & $13(4.1)$ \\
\hline Papillary & $7(2.2)$ \\
\hline Metaplastic & $4(1.3)$ \\
\hline Others & $13(4.1)$ \\
\hline \multicolumn{2}{|l|}{ T stage } \\
\hline 1 & $194(61.4)$ \\
\hline 2 & $113(35.8)$ \\
\hline 3 & $8(2.5)$ \\
\hline 4 & $1(0.3)$ \\
\hline \multicolumn{2}{|l|}{ N stage } \\
\hline 0 & $212(67.1)$ \\
\hline 1 & $77(24.4)$ \\
\hline 2 & $19(6.0)$ \\
\hline 3 & $8(2.5)$ \\
\hline \multicolumn{2}{|l|}{ ALN metastasis } \\
\hline Positive & $101(32.0)$ \\
\hline Negative & $215(68.0)$ \\
\hline \multicolumn{2}{|l|}{ FDG-avid SUV (breast) } \\
\hline Positive & $108(34.2)$ \\
\hline Negative & $208(65.8)$ \\
\hline SUV uptake max (breast)* & $3.90 \pm 3.40(0-26.6)$ \\
\hline SUV uptake max (axilla) & $0.81 \pm 2.26(0-21.9)$ \\
\hline Total patients & $316(100)$ \\
\hline
\end{tabular}

$\mathrm{BMI}=$ body mass index; IDC = invasive ductal carcinoma; ILC = invasive lobular carcinoma; $\mathrm{ALN}=$ axillary lymph node; $\mathrm{FDG}={ }^{18} \mathrm{~F}$-fluorodeoxyglucose; $\mathrm{SUV}=$ standardized uptake value.

*Mean \pm SD (range). 


\section{Statistical analyses}

Receiver operating characteristic (ROC) analysis was conducted to determine the abilities of the $\mathrm{SUV}_{\mathrm{LN} / \mathrm{T}}$ and breast MRI scans to detect involvement of $\geq 3$ ALNs. The optimal SUV $V_{\mathrm{LN} / \mathrm{T}}$ that offered the highest sum of sensitivity and specificity for detecting ALN metastasis was determined. The sensitivity, specificity, positive predictive value, and negative predictive value of both the $\mathrm{SUV}_{\mathrm{LN} / \mathrm{T}}$ and breast MRI were then calculated. Diagnostic performances were compared using diagnostic odds ratio (DOR) and area under the curve (AUC) from ROC analysis. Statistical analyses were performed using SPSS version 23.0 (IBM Corp., Armonk, USA). Two-tailed $p$-values < 0.05 were considered statistically significant.

In a subgroup of patients who showed FDG-avid primary tumors (divided by the mean SUV of the primary breast tumor), predictability of the $S U V_{\mathrm{LN} / \mathrm{T}}$ and MRI scan was assessed by ROC curves, sensitivity, specificity, and DOR. The Differences between FDG-avid and nonavid patient groups were evaluated using Student t-test, the chisquare test, or Fisher exact test.

\section{RESULTS}

A total of 316 women (mean age, $55.1 \pm 11.8$ years) who had been diagnosed with breast cancer were included in this study. The demo- graphic characteristics of the patients are presented in Table 1.

The histology for $85 \%$ of patients was invasive ductal carcinoma. ALN metastases were histologically confirmed by ALND or SLN biopsy. The flow chart of axillary treatment in our institute is shown in Figure 1. Of the 316 study patients, 36 (11.4\%) showed involvement of $\geq 3$ ALNs, and 101 (32\%) had at least one metastatic LN.

Axillary FDG uptake was positive in 75 (23.7\%) of the study patients. The mean SUV of the primary tumor was 3.9 (range, 0.0-26.6), while the mean SUV of the ALNs was 0.81 (range, 0.0-21.9). In 23 patients (7.3\%), the primary tumor showed no FDG uptake. Among these patients, 21 demonstrated no FDG uptake, but two patients showed FDG uptake only in ALNs. We excluded the $S U V_{\mathrm{LN} / \mathrm{T}}$ results of these 23 patients because they are incalculable. Based on ROC curve analysis, an optimal $\mathrm{SUV}_{\mathrm{LN} / \mathrm{T}}$ cutoff point of 0.3 was determined for predicting the necessity of ALND ( $\geq 3$ ALN metastases).

Suspicious ALN involvement was observed on MRI in 147 patients (46.6\%). The sensitivity and specificity of MRI for predicting the necessity of ALND were $88.9 \%$ and $56.2 \%$, respectively, while those of $\mathrm{SUV}_{\mathrm{LN} / \mathrm{T}}$ were $69.4 \%$ and $86.8 \%$. AUCs for MRI scans and $\mathrm{SUV}_{\mathrm{LN} / \mathrm{T}}$ were 0.756 (95\% confidence interval [CI], 0.682-0.829) and 0.817 (95\% CI, 0.733-0.900), respectively. Further analysis of DOR determined values of 10.37 and 9.7 for MRI scans and $\mathrm{SUV}_{\mathrm{LN} / \mathrm{T}}$, respectively.

However, in a subgroup of patients with FDG-avid primary tumors

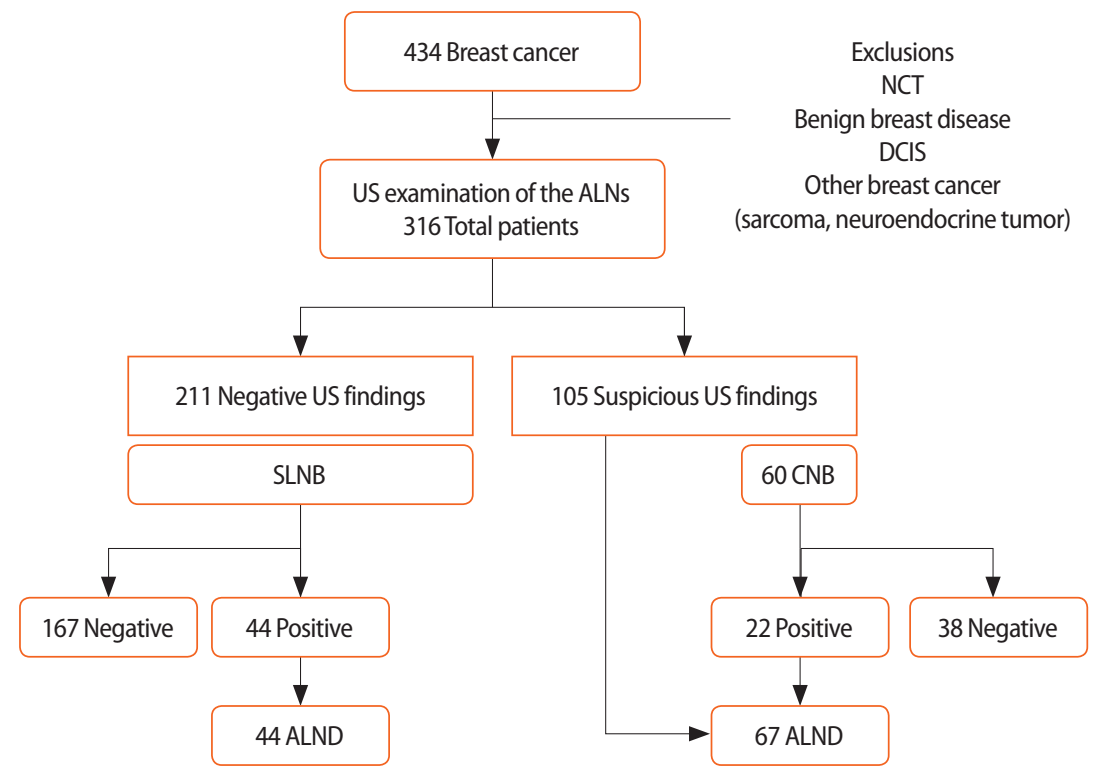

Figure 1. Flow chart of axillary treatment.

$\mathrm{NCT}=$ neoadjuvant chemotherapy; $\mathrm{DCIS}=$ ductal carcinoma in situ; US=ultrasonography; $\mathrm{ALN}=$ axillary lymph node; SLNB=sentinel lymph node biopsy; $C N B=$ core needle biopsy; ALND=axillary lymph node dissection. 


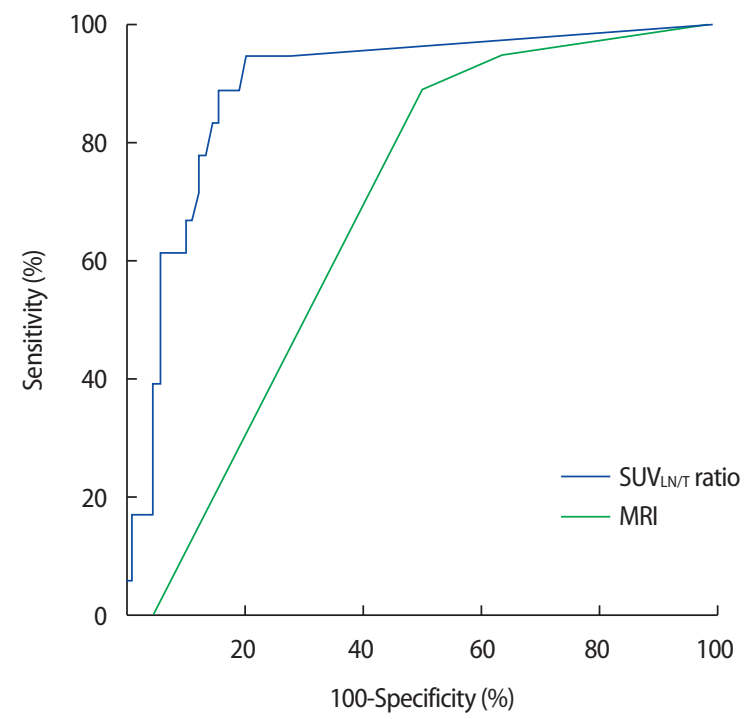

Figure 2. Receiver operating characteristic curves for SUV $V_{L N / T}$ ratio in a subgroup with FDG-avid breast tumors.

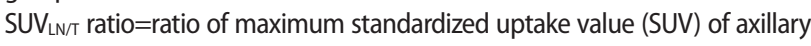
lymph node and primary tumor; $\mathrm{MRI}=$ magnetic resonance imaging; $\mathrm{FDG}={ }^{18} \mathrm{~F}$-fluorodeoxyglucose.

(FDG uptake > 3.9, $\mathrm{n}=108)$, the AUC improved to 0.896 (95\% CI, 0.817-0.975) for SUV $\mathrm{LN}_{\mathrm{L} / \mathrm{T}}$, while the AUC of MRI scans decreased to 0.681 (95\% CI, 0.569-0.793). The DOR, sensitivity, and specificity of the $\mathrm{SUV}_{\mathrm{LN} / \mathrm{T}}$ for FDG-avid tumors was $25.68,89.0 \%$ and $86.0 \%$, respectively (Figure 2). Based on ROC curve analysis, the optimal cutoff point of $\mathrm{SUV}_{\mathrm{LN} / \mathrm{T}}$ was 0.27 for predicting the necessity of $\mathrm{ALND}(\geq 3$ ALN metastases). Diagnostic performance of the $\mathrm{SUV}_{\mathrm{LN} / \mathrm{T}}$ ratio and MRI are shown in Table 2.

We also compared FDG avidity and certain patient characteristics, which as shown in Table 3. A higher SUV for primary breast tumors correlated with multiple prognostic variables, including tumor size, estrogen receptor (ER) negativity, and progesterone receptor (PR) negativity.

\section{DISCUSSION}

The aim of the present study was to assess the predictive value of the LN to tumor SUV ratio in patients with breast cancer. We compared MRI scans and FDG-uptake, from PET/CT imaging, for the detection of three or more axillary metastases. Our study showed that the SUV $V_{\mathrm{LN} / \mathrm{T}}$ could accurately predict the necessity of ALND dissection for patients with FDG-avid primary breast cancer.
Table 2. Diagnostic performance of SUV $V_{\text {LN/T }}$ ratio and MRI

\begin{tabular}{lccccc}
\hline & $\begin{array}{c}\text { Sensitivity } \\
(\%)\end{array}$ & $\begin{array}{c}\text { Specificity } \\
(\%)\end{array}$ & PPV & NPV & DOR \\
\hline All tumors & & & & & \\
FDG-SUV ratio & 69.4 & 86.8 & 40.3 & 95.7 & 9.7 \\
$\quad$ MRI & 88.9 & 56.2 & 21.8 & 97.5 & 10.37 \\
$\quad \begin{array}{l}\text { Tumors with FDG }>3.9 \\
\quad \text { FDG-SUV ratio }\end{array}$ & 89.0 & 86.0 & 51.7 & 96.2 & 25.68 \\
MRI & 94.4 & 38.4 & 24.3 & 97.1 & 9.61 \\
\hline
\end{tabular}

SUV $V_{\mathrm{LN} / \mathrm{T}}$ ratio = ratio of maximum standardized uptake value (SUV) of axillary lymph node and primary tumor; $M R I=$ magnetic resonance imaging; $\mathrm{PPV}=$ positive predictive value; $\mathrm{NPV}=$ negative predictive value; $\mathrm{DOR}=$ diagnostic odds ratio; $\mathrm{FDG}={ }^{18} \mathrm{~F}$-fluorodeoxyglucose.

Table 3. Characteristics of breast cancer patients categorized according to FDG avidity of primary tumor

\begin{tabular}{lccc}
\hline & \multicolumn{2}{c}{ FDG } & \\
\cline { 2 - 3 } Patient factor & $\begin{array}{c}\geq 3.9 \\
\text { No. }(\%)\end{array}$ & $\begin{array}{c}<3.9 \\
\text { No. }(\%)\end{array}$ & \\
\hline Age $(\mathrm{yr})^{*}$ & $56.19 \pm 12.9$ & $54.37 \pm 10.7$ & 0.186 \\
Body mass index* & $24 \pm 3.3$ & $24.4 \pm 4.0$ & 0.435 \\
Tumor $(\mathrm{cm})$ & & & $<0.001$ \\
$\leq 2$ & 37 & 153 & \\
$>2$ & 71 & 55 & \\
Estrogen receptor & & & $<0.001$ \\
$\quad$ Positive & $63(58.3)$ & $168(80.8)$ & \\
$\quad$ Negative & $45(41.7)$ & $40(19.2)$ & \\
Progesterone receptor & & & $<0.001$ \\
$\quad$ Positive & $51(47.7)$ & $155(74.5)$ & \\
$\quad$ Negative & $56(52.3)$ & $53(25.5)$ & \\
HER2 & & & 0.367 \\
$\quad$ Overexpression & $98(90.7)$ & $177(85.1)$ & \\
$\quad$ Non-overexpression & $10(9.3)$ & $31(14.9)$ & \\
Total & $108(100)$ & $208(100)$ & \\
\hline
\end{tabular}

$\mathrm{FDG}={ }^{18} \mathrm{~F}$-fluorodeoxyglucose; HER2 = human epidermal growth factor receptor 2 .

*Mean \pm SD.

Advances in preoperative imaging have advanced and optimized axillary management [11]. For minimally invasive breast cancer surgery, preoperative evaluation of the ALNs is important. There have been a few reported studies comparing MRI and PET/CT for the diagnosis of LN metastasis. Recently, Liang et al. [12] reported a meta-analysis that suggested MRI scans had higher sensitivity than PET/ CT imaging (0.82 vs. 0.64) for the diagnosis of ALN metastasis in patients with breast cancer.

For distinguishing the involvement of at least one LN, PET parameters showed limited diagnostic performance, attributed to the partial volume effect, relatively low FDG-uptake by low-grade malignancies, 
and FDG-uptake by benign entities [13,14]. Nonetheless, FDG-PET provides metabolic information, and hence, combination of the FDGPET with other imaging modalities may improve the diagnostic accuracy for detecting ALN metastasis in breast cancer [15]. In addition, $\mathrm{SUV}_{\mathrm{LN} / \mathrm{T}}$ might be a more objective diagnostic method than axillary ultrasonography, which is operator-dependent, for predicting LN metastasis.

Previous studies showed that the accuracy of $S U V_{\mathrm{LN} / \mathrm{T}}$ for predicting the presence of ALN metastasis was superior to that of SUV $\mathrm{LN}_{\mathrm{LN}}[15]$. This finding may result from variation in SUV measurements from multiple hospital protocols, as well as the influence of factors including body composition, blood glucose levels, length of the uptake period, and the partial volume effect. In addition, $\mathrm{SUV}_{\mathrm{LN}}$ is related to the FDG avidity of the primary tumor $[16,17]$. Therefore, the axillary $S_{U} V_{\mathrm{LN} / \mathrm{T}}$ might predict LN status more objectively than nodal SUV alone.

An important finding of the present study is that $\mathrm{SUV}_{\mathrm{LN} / \mathrm{T}}$ appears to be particularly reliable in patients with an $\mathrm{SUV}_{\mathrm{T}}$ greater than 3.9. Therefore, tumors with a low SUV, including low-grade malignancies or tumors combined with benign inflammatory lesions, warrant additional morphological evaluation by conventional modalities. Higher SUV for primary breast tumors in our study correlated with tumor size, ER negativity, and PR negativity. Furthermore, Peterson et al. [18] and Gemignani et al. [19] found an association with body mass index, sex hormone-binding globulin, and PR expression in ${ }^{18} \mathrm{~F}$-FES PET uptake.

In the present study, the specificity for predicting three or more ALN metastases using $\mathrm{SUV}_{\mathrm{LN} / \mathrm{T}}$ was much higher than the specificity achieved using MRI. To achieve a higher specificity, SLN biopsy might be an optional but nonessential procedure in patients with highly suspicious ALN metastasis, as determined by PET/CT imaging; these patients could undergo complete ALND as the primary procedure, an approach that would reduce time and cost.

The limitations of the present study include the possibility of inherent biases associated with a retrospective study design, as well as the small sample size. In addition, PET/CT scans were interpreted by a single, albeit experienced, nuclear medicine physician.

Axillary LN evaluation is crucial for staging and therapeutic planning in patients with invasive breast cancer. Although the current data were retrospectively analyzed at a single institution, our findings suggest that $\mathrm{SUV}_{\mathrm{LN} / \mathrm{T}}$, as obtained by PET/CT, may help predict the presence of three or more ALN metastases in an FDG-avid subgroup prior to surgery. A large, prospective cohort study may be recommended to validate $S U V_{\mathrm{LN} / \mathrm{T}}$ as a reliable predictor of three or more metastases in the ALNs.

In conclusion, $\mathrm{SUV}_{\mathrm{LN} / \mathrm{T}}$ can identify the presence of three or more ALN metastases, particularly in patients with FDG-avid breast tumors. PET/CT imaging may be a valuable tool for treatment selection in advanced breast cancer.

\section{CONFLICT OF INTEREST}

The authors declare that they have no competing interests.

\section{REFERENCES}

1. Giuliano AE, Hunt KK, Ballman KV, Beitsch PD, Whitworth PW, Blumencranz PW, et al. Axillary dissection vs no axillary dissection in women with invasive breast cancer and sentinel node metastasis: a randomized clinical trial. JAMA 2011;305:569-75.

2. Chao C, Wong SL, Ackermann D, Simpson D, Carter MB, Brown $\mathrm{CM}$, et al. Utility of intraoperative frozen section analysis of sentinel lymph nodes in breast cancer. Am J Surg 2001;182:609-15.

3. Caretta-Weyer H, Sisney GA, Beckman C, Burnside ES, Salkowsi LR, Strigel RM, et al. Impact of axillary ultrasound and core needle biopsy on the utility of intraoperative frozen section analysis and treatment decision making in women with invasive breast cancer. Am J Surg 2012;204:308-14.

4. Luciani A, Itti E, Rahmouni A, Meignan M, Clement O. Lymph node imaging: basic principles. Eur J Radiol 2006;58:338-44.

5. Yang SK, Cho N, Moon WK. The role of PET/CT for evaluating breast cancer. Korean J Radiol 2007;8:429-37.

6. Liu Y. Role of FDG PET-CT in evaluation of locoregional nodal disease for initial staging of breast cancer. World J Clin Oncol 2014; 5:982-9.

7. Machida Y, Kubota K, Katayama T, Toriihara A, Shibuya H. Diagnostic performance of fluorodeoxyglucose-positron emission tomography/computed tomography combined with ultrasonography-guided fine needle aspiration cytology for identifying axillary lymph node status in patients with breast cancer. Eur J Surg Oncol 2013;39:26-30. 
8. Chae BJ, Bae JS, Kang BJ, Kim SH, Jung SS, Song BJ. Positron emission tomography-computed tomography in the detection of axillary lymph node metastasis in patients with early stage breast cancer. Jpn J Clin Oncol 2009;39:284-9.

9. Kim BS, Sung SH. Usefulness of 18F-FDG uptake with clinicopathologic and immunohistochemical prognostic factors in breast cancer. Ann Nucl Med 2012;26:175-83.

10. Choi WH, Yoo IR, O JH, Kim SH, Chung SK. The value of dualtime-point 18F-FDG PET/CT for identifying axillary lymph node metastasis in breast cancer patients. Br J Radiol 201 1;84:593-9.

11. Ahn SK, Kim MK, Kim J, Lee E, Yoo TK, Lee HB, et al. Can we skip intraoperative evaluation of sentinel lymph nodes? Nomogram predicting involvement of three or more axillary lymph nodes before breast cancer surgery. Cancer Res Treat 2017;49:1088-96.

12. Liang X, Yu J, Wen B, Xie J, Cai Q, Yang Q. MRI and FDG-PET/CT based assessment of axillary lymph node metastasis in early breast cancer: a meta-analysis. Clin Radiol 2017;72:295-301.

13. Soret M, Bacharach SL, Buvat I. Partial-volume effect in PET tumor imaging. J Nucl Med 2007;48:932-45.

14. Lim HS, Yoon W, Chung TW, Kim JK, Park JG, Kang HK, et al. FDG PET/CT for the detection and evaluation of breast diseases: usefulness and limitations. Radiographics 2007;27 Suppl 1:S197-
213.

15. Park J, Byun BH, Noh WC, Lee SS, Kim HA, Kim EK, et al. Lymph node to primary tumor SUV ratio by 18 F-FDG PET/CT and the prediction of axillary lymph node metastases in breast cancer. Clin Nucl Med 2014;39:e249-53.

16. Lovrics PJ, Chen V, Coates G, Cornacchi SD, Goldsmith CH, Law C, et al. A prospective evaluation of positron emission tomography scanning, sentinel lymph node biopsy, and standard axillary dissection for axillary staging in patients with early stage breast cancer. Ann Surg Oncol 2004;11:846-53.

17. Ahn JH, Son EJ, Kim JA, Youk JH, Kim EK, Kwak JY, et al. The role of ultrasonography and FDG-PET in axillary lymph node staging of breast cancer. Acta Radiol 2010;51:859-65.

18. Peterson LM, Kurland BF, Link JM, Schubert EK, Stekhova S, Linden HM, et al. Factors influencing the uptake of 18F-fluoroestradiol in patients with estrogen receptor positive breast cancer. Nucl Med Biol 2011;38:969-78.

19. Gemignani ML, Patil S, Seshan VE, Sampson M, Humm JL, Lewis JS, et al. Feasibility and predictability of perioperative PET and estrogen receptor ligand in patients with invasive breast cancer. J Nucl Med 2013;54:1697-702. 